Nitrogen fixation commonly occurs in bacteria only when the oxygen tension is low either because the nitrogen fixer is an obligate anaerobe such as Clostridium or because the aerobic organism, Azotobacter for example, has in addition to the normal ATP synthesising system, a very active electron transport chain that operates without conserving energy. This results in a microaerobic environment around the site of nitrogen fixation (Postgate, in Biological nitrogen fixation, edit. by Quispel, A., 1974) and brings about a respiratory protection of the nitrogenase.

The nitrogen-fixing blue-green algae have a particular problem since their photosynthesis leads to oxygen evolution; they may fix nitrogen only at low oxygen tensions and low light intensity (non-heterocystous forms) or they may segregate their nitrogenase into special compartments (the heterocysts) to which the fixation of nitrogen is normally confined. Only under artificial conditions of anaerobiosis do the ordinary vegetative cells of heterocystous blue greens have active nitrogenase.

Heterocysts are specialised in that they seem to lack the oxygenevolving component of photosyn- thesis, photosystem 2. They may also remove unwanted oxygen diffusing in from the environment by the use of an active respiratory chain. Thus a low oxygen tension could be maintained at the site of nitrogen fixation.

The absence of photosystem 2 has been deduced from the lack of accessory pigments, from the low yields of chlorophyll fluorescence and from the inability of heterocysts to fix carbon dioxide (Stewart, A. Rev.

\section{Nitrogen, oxygen and manganese} from $F$. R. Whatley

Microbiol., 27, 283; 1973). The association of photosystem 2 with manganese has also been widely documented (for a review see Cheniae, A. Rev. Pl. Physiol., 21, 467; 1970) although the evidence has always been indirect. Recently Tel-Or and Avron (Proc. third int. Congress on Photosynthesis, 569; 1974) reported isolation of a heat stable manganesecontaining compound of small molecular weight which, when added back to depleted particles isolated from a blue-green alga (Phormidium luridum) restored oxygen evolving ability, thus providing direct evidence of a role for manganese in $\mathrm{O}_{2}$ evolution.

The lack of photosystem 2 in heterocysts in another blue-green alga (Anabaena cylindrica) is now further substantiated (Tel-Or and Stewart, Nature, 258, 715; 1975), following an examination of the distribution of ${ }^{54} \mathrm{Mn}$ between vegetative cells and heterocysts isolated from cultures grown with ${ }^{54} \mathrm{Mn}$. This showed a correlation between the presence of ${ }^{54} \mathrm{Mn}$, the $\mathrm{Mn}$ : chlorophyll ratio, and the ability of the isolated cells to evolve oxygen. The clear conclusion is that heterocysts of these nitrogen-fixing algae are deficient in manganese, that they lack a functional photosystem 2 and therefore cannot evolve oxygen. Heterocysts are thus specially adapted for nitrogen fixation. By contrast, the vegetative cells accumulate manganese and evolve oxygen but do not appear to fix nitrogen. It is of correlative interest (Anderson et al. Biochem. biophys. Res. Commun., $17,685 ; 1974)$ that fractions of isolated chloroplasts which have been depleted in photosystem 2 also become depleted in bound manganese whereas those enriched in photosystem 2 become enriched in manganese. female's eggs by dashing into a territory at the last moment. More often, they chase an arriving female as a group and achieve a sort of piscine gang bang.

The facts are remarkable enough, but what is the evolutionary explanation for this strange reproductive system? Warner, Robertson and Leigh (Science, 190, 633-638; 1975) develop an idea of M. T. Ghiselin, who argued that natural selection will favour sex change whenever the reproductive potential of one sex increases rapidly with age while the other remains fairly constant. In this circumstance it will be advantageous to start life as the sex which does not benefit from being older (or to put it another way, does not suffer from being young) and switch to the other sex at a later stage. This is what seems to be happening in the Blue-headed Wrasse. Terminal males have an enormous reproductive potential, while initial males have a low mating success, so an individual can get the best of both worlds, by starting off as a female (young females do not suffer any penalty for youth) and switch to being a male at a later stage when it can successfully defend a mating territory. But why do some individuals go through the initial phase as a male and not a female? Warner et al. suggest that in large populations, where many females arrive simul- taneously at the spawning area, the opportunities for an initial phase male to grab the odd fertilisation are high enough to make being a male in early life about as profitable as being a female. Their data show that the daily mating rates of an initial male and a female in a large population are about the same. In a small population, however, there are fewer chances for the initial males. There are rarely enough females at the spawning site to enable an initial male to steal a fertilisation. As this account would predict, initial males are much rarer in small populations than in large ones. Also, in other wrasse species that always occur in small groups, initial phase males are always rare.

If, as Warner et al. suggest, sex change is an optimal strategy when there is a big advantage in being old in one sex but not in the other, why don't mammals such as seals, deer and baboons, in which the older males monopolise females, change sex like the wrasse? The authors suggest that one or more of three factors may prevent them from doing so: the physiological cost of changing sex in an animal with internal fertilisation, the rigidity of the mammalian sex determination system, and the fact that in mammals competitive experience as well as size is important in determining the reproductive superi- ority of older males. Obviously this experience would be lost if a male spent its youth as a member of the opposite sex.

\section{How old are cosmic rays?}

from J. J. Quenby

WHILE recent progress in observational astrophysics has been rapid, the answer to the fundamental question as to the origin of cosmic radiation remains elusive. This flux of relativistic, charged particles populates the Galaxy with an energy density sufficient to influence significantly the dynamics of the whole system, but we do not know whether relatively near objects like the Crab Nebula or distant, energetic objects like strong radio galaxies constitute the prime source. An important clue would lie in an ability to 'read' the ${ }^{10} \mathrm{Be}$ 'clock' in the cosmic ray flux. The light elements $\mathrm{Li}, \mathrm{Be}$ and $\mathrm{B}$ are rare in cosmic material and should not be present at the source of particle acceleration. By knowing production cross sections for the light elements in collisions between nucleons and atoms it is possible to calculate the amount of material through which the average 